Commun. Fac. Sci. Univ. Ank. Ser. A1 Math. Stat.

Volume 69, Number 1, Pages 547 559(2020)

DOI: $10.31801 /$ cfsuasmas. 527107

ISSN 1303-5991 E-ISSN 2618-6470

http://communications.science.ankara.edu.tr/index.php?series=A1

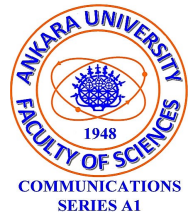

\title{
DECOMPOSITION OF SOFT CONTINUITY VIA SOFT LOCALLY $b$-CLOSED SET
}

\author{
NAİME DEMİRTAŞ AND ZEHRA GÜZEL ERGÜL
}

\begin{abstract}
In this paper, we introduce soft locally $b$-closed sets in soft topological spaces which are defined over an initial universe with a fixed set of parameters and study some of their properties. We investigate their relationships with different types of subsets of soft topological spaces with the help of counterexamples. Also, the concept of soft locally $b$-continuous functions is presented. Finally, a decomposition of soft continuity is obtained.
\end{abstract}

\section{INTRODUCTION}

Molodtsov 18, 19 initiated and applied soft set theory, while modelling the problems in the field of science including engineering physics, computer science, economics, social sciences and medical sciences, to deal with uncertain data and not clear objects without complete information. Then many researchers [9, 16, 17, 20, 24 presented some new definitions and results and also discussed in detail the application of soft set theory in decision making problems.

In [20], Shabir and Naz introduced the primary concepts of soft topological spaces. Later, Aygünoğlu and Aygün [7], Hussain and Ahmad [11, Yuksel et al. 23. Zorlutuna et al. 24. continued to study many basic concepts and properties of soft topological spaces. Kharal and Ahmad [13 and Zorlutuna et al. 24] discussed the mappings of soft classes and their properties in soft topological spaces. Recently, different forms of soft open sets [1, 2, 3, 6, 8, 12, 14, 15, 21, were studied.

In the present paper, we introduce soft locally $b$-closed set in soft topological spaces which are defined over an initial universe with a fixed set of parameters. Also, we give the notion of soft locally $b$-continuous function and obtain another decomposition of soft continuity.

Received by the editors: February 14, 2019; Accepted: December 02, 2019.

2010 Mathematics Subject Classification. Primary 54A05; Secondary 54A40, 03E72.

Key words and phrases. Soft set, soft topological space, soft locally b-closed set, soft locally $b$-continuous function.

(C)2020 Ankara University Communications Faculty of Sciences University of Ankara-Series A1 Mathematics and Statistics 


\section{Preliminaries}

Here, we present the basic definitions and results of soft sets and soft topological spaces which have already given in earlier studies. Let $X$ be an initial universe set and $E$ be the set of all possible parameters with respect to $X$. Let $P(X)$ denote the power set of $X$. Then a soft set over $X$ is defined as follows.

Definition 1. 18, A pair $(F, A)$ is called a soft set over $X$ where $A \subseteq E$ and $F: A \rightarrow P(X)$ is a set valued mapping. In other words, a soft set over $X$ is a parameterized family of subsets of the universe $X$. For $\forall \varepsilon \in A, F(\varepsilon)$ may be considered as the set of $\varepsilon$-approximate elements of the soft set $(F, A)$. It is worth noting that $F(\varepsilon)$ may be arbitrary. Some of them may be empty, and some may have nonempty intersection.

The set of all soft sets over $X$ is denoted by $S S(X)_{E}$. For null soft set $(\Phi)$, absolute soft set $(\tilde{X})$, soft subset $(\sqsubseteq)$, soft union $(\sqcup)$, soft intersection $(\sqcap)$, soft relative complement, their properties and the relations to each other; the interested reader is refer to [5, 9, 17, 20, 24].

Definition 2. 24] The soft set $(F, E) \in S S(X)_{E}$ is called a soft point in $\tilde{X}$, denoted by $e_{F}$, if for the element $e \in E, F(e) \neq \emptyset$ and $F\left(e^{\prime}\right)=\emptyset$ for all $e^{\prime} \in E \backslash\{e\}$. The soft point $e_{F}$ is said to be in the soft set $(G, E)$, denoted by $e_{F} \in(G, E)$, if for the element $e \in E$ and $F(e) \subseteq G(e)$.

Definition 3. 20] Let $\tilde{\tau}$ be the collection of soft sets over $X$, then $\tilde{\tau}$ is said to be a soft topology on $X$ if

(1) $\Phi, \tilde{X}$ belong to $\tilde{\tau}$

(2) the union of any number of soft sets in $\tilde{\tau}$ belongs to $\tilde{\tau}$,

(3) the intersection of any two soft sets in $\tilde{\tau}$ belongs to $\tilde{\tau}$.

The triplet $(X, \tilde{\tau}, E)$ is called a soft topological space over $X$. The members of $\tilde{\tau}$ are said to be soft open sets in $X$. A soft set $(F, E)$ over $X$ is said to be a soft closed set in $X$, if its relative complement $(F, E)^{c}$ belongs to $\tilde{\tau}$. We will denote the family of all soft open sets (resp., soft closed sets) of a soft topological space $(X, \tilde{\tau}, E)$ by $\operatorname{SOS}(X)$ (resp., $\operatorname{SCS}(X))$.

Throughout the paper, the spaces $X$ and $Y$ stand for soft topological spaces with $(X, \tilde{\tau}, E)$ and $(Y, \tilde{v}, K)$ assumed unless otherwise stated.

Definition 4. Let $X$ be a soft topological space and $(F, E)$ be a soft set over $X$.

(1) 20] The soft closure of $(F, E)$ is the soft set in $X$ defined as: $c l(F, E)=\sqcap\{(G, E):(G, E)$ is soft closed and $(F, E) \sqsubseteq(G, E)\}$.

(2) 24] The soft interior of $(F, E)$ is the soft set in $X$ defined as: $\operatorname{int}(F, E)=\sqcup\{(H, E):(H, E)$ is soft open and $(H, E) \sqsubseteq(F, E)\}$.

Clearly, $\operatorname{cl}(F, E)$ is the smallest soft closed set over $X$ which contains $(F, E)$ and $\operatorname{int}(F, E)$ is the largest soft open set over $X$ which is contained in $(F, E)$. 
Definition 5. Let $X$ be a soft topological space. A soft set $(F, E)$ is called

(1) a soft semiopen set $[8]$ in $X$ if $(F, E) \sqsubseteq \operatorname{cl}(\operatorname{int}(F, E))$,

(2) a soft preopen set [6] in $X$ if $(F, E) \sqsubseteq \operatorname{int}(\operatorname{cl}(F, E))$,

(3) a soft $\alpha$-open set [1] in $X$ if $(F, E) \sqsubseteq \operatorname{int}(\operatorname{cl}(\operatorname{int}(F, E)))$,

(4) a soft $\beta$-open set 12 in $X$ if $(F, E) \sqsubseteq \operatorname{cl}(\operatorname{int}(\operatorname{cl}(F, E)))$,

(5) a soft regular open set 22] in $X$ if $(F, E)=\operatorname{int}(\operatorname{cl}(F, E))$,

(6) a soft A-set 21] in $X$ if $(F, E)=(G, E) \backslash(H, E)$, where $(G, E)$ is a soft open set and $(H, E)$ is a soft regular open set in $X$,

(7) a soft t-set 21] in $X$ if $\operatorname{int}(\operatorname{cl}(F, E))=\operatorname{int}(F, E)$,

(8) a soft B-set [21] in $X$ if $(F, E)=(G, E) \sqcap(H, E)$, where $(G, E)$ is a soft open set and $(H, E)$ is a soft t-set in $X$,

(9) a soft $b$-open (briefly; sb-open) set 2 in $X$ if $(F, E) \sqsubseteq \operatorname{int}(\operatorname{cl}(F, E)) \sqcup$ $\operatorname{cl}(\operatorname{int}(F, E))$,

(10) a soft locally closed set (briefly; soft LC-set) 14 in $X$ if $(F, E)=(G, E) \sqcap$ $(H, E)$, where $(G, E)$ is soft open and $(H, E)$ is soft closed in $X$.

The relative complement of a soft semiopen (soft preopen, soft $\alpha$-open, soft $\beta$ open, soft regular open, soft $b$-open) set is called a soft semiclosed (soft preclosed, soft $\alpha$-closed, soft $\beta$-closed, soft regular closed, soft $b$-closed) set. We will denote the family of all soft semiopen sets (resp., soft preopen sets, soft $\alpha$-open sets, soft $\beta$-open sets, soft regular open sets, soft A-sets, soft B-sets, soft $b$-open sets and soft locally closed sets) of a soft topological space $X$ by $\operatorname{SSOS}(X)$ (resp., $\operatorname{SPOS}(X)$, $\operatorname{S} \alpha \operatorname{OS}(X), \operatorname{S} \beta O S(X), \operatorname{SROS}(X), \operatorname{SAS}(X), \operatorname{SBS}(X), \operatorname{SbOS}(X)$ and $\operatorname{SLCS}(X))$.

Definition 6. Let $X$ be a soft topological space and $(F, E)$ be a soft set over $X$.

(1) The soft semiclosure 8 of $(F, E)$ is the soft set in $X$ defined as: $\operatorname{scl}(F, E)=\sqcap\{(G, E):(G, E)$ is soft semiclosed and $(F, E) \sqsubseteq(G, E)\}$.

(2) The soft semiinterior $[8$ of $(F, E)$ is the soft set in $X$ defined as: $\operatorname{sint}(F, E)=\sqcup\{(H, E):(H, E)$ is soft semiopen and $(H, E) \sqsubseteq(F, E)\}$.

(3) The soft preclosure [3] of $(F, E)$ is the soft set in $X$ defined as: $\operatorname{pcl}(F, E)=\sqcap\{(G, E):(G, E)$ is soft preclosed and $(F, E) \sqsubseteq(G, E)\}$.

(4) The soft preinterior [3] of $(F, E)$ is the soft set in $X$ defined as: $\operatorname{pint}(F, E)=\sqcup\{(H, E):(H, E)$ is soft preopen and $(H, E) \sqsubseteq(F, E)\}$.

(5) The soft $b$-closure [2] of $(F, E)$ is the soft set in $X$ defined as: $b c l(F, E)=\sqcap\{(G, E):(G, E)$ is soft $b$-closed and $(F, E) \sqsubseteq(G, E)\}$. (6) The soft $b$-interior [2] of $(F, E)$ is the soft set in $X$ defined as: $\operatorname{bint}(F, E)=\sqcup\{(H, E):(H, E)$ is soft $b$-open and $(H, E) \sqsubseteq(F, E)\}$.

Theorem 7. 21] Let $X$ be a soft topological space. A soft set $(F, E)$ over $X$ is soft open if and only if it is both a soft preopen set and a soft B-set.

Proof. Necessity is trivial, we prove the sufficiency. Since $(F, E)$ is a soft B-set, we have $(F, E)=(G, E) \sqcap(H, E)$, where $(G, E)$ is a soft open set and $\operatorname{int}(\operatorname{cl}(H, E))=$ 
$\operatorname{int}(H, E)$. Since $(F, E)$ is soft preopen, we have

$$
\begin{aligned}
(F, E) & \sqsubseteq \operatorname{int}(\operatorname{cl}(F, E))=\operatorname{int}(\operatorname{cl}((G, E) \sqcap(H, E))) \\
& \sqsubseteq \operatorname{int}(\operatorname{cl}(G, E) \sqcap \operatorname{cl}(H, E))=\operatorname{int}(\operatorname{cl}(G, E)) \sqcap \operatorname{int}(\operatorname{cl}(H, E)) \\
& =\operatorname{int}(\operatorname{cl}(G, E)) \sqcap \operatorname{int}(H, E) .
\end{aligned}
$$

Hence

$$
\begin{aligned}
(F, E) & =(G, E) \sqcap(H, E)=((G, E) \sqcap(H, E)) \sqcap(G, E) \\
& \sqsubseteq(\operatorname{int}(\operatorname{cl}(G, E)) \sqcap \operatorname{int}(H, E)) \sqcap(G, E) \\
& =(\operatorname{int}(\operatorname{cl}(G, E)) \sqcap(G, E)) \sqcap \operatorname{int}(H, E)=(G, E) \sqcap \operatorname{int}(H, E) .
\end{aligned}
$$

Notice $(F, E)=(G, E) \sqcap(H, E) \sqsupseteq(G, E) \sqcap \operatorname{int}(H, E)$, we have $(F, E)=(G, E) \sqcap$ $\operatorname{int}(H, E)$. Thus we obtain $(F, E)$ is soft open.

Theorem 8. [12] Let $X$ be a soft topological space. A soft set $(F, E)$ over $X$ is soft $\alpha$-open if and only if it is both a soft preopen set and a soft semiopen set.

Proof. Necessity. Since $(F, E)$ is a soft $\alpha$-open set, we have $(F, E) \sqsubseteq \operatorname{int}(\operatorname{cl}(\operatorname{int}(F, E)))$. Hence $(F, E) \sqsubseteq \operatorname{cl}(\operatorname{int}(F, E))$ and $(F, E) \sqsubseteq \operatorname{int}(\operatorname{cl}(F, E))$. Thus $(F, E)$ is both soft preopen and soft semiopen.

Sufficiency. Since $(F, E)$ is both a soft preopen set and a soft semiopen set, then $(F, E) \sqsubseteq \operatorname{cl}(\operatorname{int}(F, E))$ and $(F, E) \sqsubseteq \operatorname{int}(\operatorname{cl}(F, E))$. Thus

$$
(F, E) \sqsubseteq \operatorname{int}(\operatorname{cl}(\operatorname{cl}(\operatorname{int}(F, E))))=\operatorname{int}(\operatorname{cl}(\operatorname{int}(F, E))) .
$$

It follows that $(F, E)$ is soft $\alpha$-open.

\section{Soft Locally b-Closed Sets}

In this section, we introduce soft locally $b$-closed sets in soft topological spaces and study some of their properties.

Definition 9. A soft set $(F, E)$ in a soft topological space $X$ is called a soft locally b-closed set (briefly; soft LbC-set) if $(F, E)=(G, E) \sqcap(K, E)$ where $(G, E)$ is soft open and $(K, E)$ is soft b-closed.

Remark 10. The following examples show that a soft locally $b$-closed set need not be soft open and a soft locally b-closed set need not be soft b-closed.

Example 11. Let $X=\left\{x_{1}, x_{2}, x_{3}, x_{4}\right\}, E=\left\{e_{1}, e_{2}, e_{3}\right\}$ and

$$
\tilde{\tau}=\left\{\Phi, \tilde{X},\left(F_{1}, E\right),\left(F_{2}, E\right), \ldots,\left(F_{15}, E\right)\right\}
$$

where $\Phi, \tilde{X},\left(F_{1}, E\right),\left(F_{2}, E\right), \ldots,\left(F_{15}, E\right)$ are soft sets over $X$, defined as follows:

$$
\begin{aligned}
& \left(F_{1}, E\right)=\left\{\left(e_{1},\left\{x_{1}\right\}\right),\left(e_{2},\left\{x_{2}, x_{3}\right\}\right),\left(e_{3},\left\{x_{1}, x_{4}\right\}\right)\right\} \\
& \left(F_{2}, E\right)=\left\{\left(e_{1},\left\{x_{2}, x_{4}\right\}\right),\left(e_{2},\left\{x_{1}, x_{3}, x_{4}\right\}\right),\left(e_{3},\left\{x_{1}, x_{2}, x_{4}\right\}\right)\right\}
\end{aligned}
$$




$$
\begin{aligned}
\left(F_{3}, E\right) & =\left\{\left(e_{1}, \emptyset\right),\left(e_{2},\left\{x_{3}\right\}\right),\left(e_{3},\left\{x_{1}\right\}\right)\right\} \\
\left(F_{4}, E\right) & =\left\{\left(e_{1},\left\{x_{1}, x_{2}, x_{4}\right\}\right),\left(e_{2}, X\right),\left(e_{3}, X\right)\right\} \\
\left(F_{5}, E\right) & =\left\{\left(e_{1},\left\{x_{1}, x_{3}\right\}\right),\left(e_{2},\left\{x_{2}, x_{4}\right\}\right),\left(e_{3},\left\{x_{2}\right\}\right)\right\} \\
\left(F_{6}, E\right) & =\left\{\left(e_{1},\left\{x_{1}\right\}\right),\left(e_{2},\left\{x_{2}\right\}\right),\left(e_{3}, \emptyset\right)\right\} \\
\left(F_{7}, E\right) & =\left\{\left(e_{1},\left\{x_{1}, x_{3}\right\}\right),\left(e_{2},\left\{x_{2}, x_{3}, x_{4}\right\}\right),\left(e_{3},\left\{x_{1}, x_{2}, x_{4}\right\}\right)\right\} \\
\left(F_{8}, E\right) & =\left\{\left(e_{1}, \emptyset\right),\left(e_{2},\left\{x_{4}\right\}\right),\left(e_{3},\left\{x_{2}\right\}\right)\right\} \\
\left(F_{9}, E\right) & =\left\{\left(e_{1}, X\right),\left(e_{2}, X\right),\left(e_{3},\left\{x_{1}, x_{2}, x_{3}\right\}\right)\right\} \\
\left(F_{10}, E\right) & =\left\{\left(e_{1},\left\{x_{1}, x_{3}\right\}\right),\left(e_{2},\left\{x_{2}, x_{3}, x_{4}\right\}\right),\left(e_{3},\left\{x_{1}, x_{2}\right\}\right)\right\} \\
\left(F_{11}, E\right) & =\left\{\left(e_{1},\left\{x_{2}, x_{3}, x_{4}\right\}\right),\left(e_{2}, X\right),\left(e_{3},\left\{x_{1}, x_{2}, x_{3}\right\}\right)\right\} \\
\left(F_{12}, E\right) & =\left\{\left(e_{1},\left\{x_{1}\right\}\right),\left(e_{2},\left\{x_{2}, x_{3}, x_{4}\right\}\right),\left(e_{3},\left\{x_{1}, x_{2}, x_{4}\right\}\right)\right\} \\
\left(F_{13}, E\right) & =\left\{\left(e_{1},\left\{x_{1}\right\}\right),\left(e_{2},\left\{x_{2}, x_{4}\right\}\right),\left(e_{3},\left\{x_{2}\right\}\right)\right\} \\
\left(F_{14}, E\right) & =\left\{\left(e_{1},\left\{x_{3}, x_{4}\right\}\right),\left(e_{2},\left\{x_{1}, x_{2}\right\}\right),\left(e_{3}, \emptyset\right)\right\} \\
\left(F_{15}, E\right) & =\left\{\left(e_{1},\left\{x_{1}\right\}\right),\left(e_{2},\left\{x_{2}, x_{3}\right\}\right),\left(e_{3},\left\{x_{1}\right\}\right)\right\}
\end{aligned}
$$

Then $\tilde{\tau}$ defines a soft topology on $X$ and thus $(X, \tilde{\tau}, E)$ is a soft topological space over $X$ in [2]. Let $(H, E)$ be a soft $b$-closed set over $X$ such that $(H, E)=$ $\left\{\left(e_{1},\left\{x_{1}, x_{2}, x_{3}\right\}\right),\left(e_{2},\left\{x_{4}\right\}\right),\left(e_{3},\left\{x_{1}, x_{3}\right\}\right)\right\}$. Then $\left(F_{8}, E\right) \sqcap(H, E)=(F, E)=$ $\left\{\left(e_{1}, \emptyset\right),\left(e_{2},\left\{x_{4}\right\}\right),\left(e_{3}, \emptyset\right)\right\}$ is a soft locally $b$-closed set in $X$, but $(F, E)$ is not soft open.

Example 12. Let $X=\left\{x_{1}, x_{2}, x_{3}, x_{4}\right\}, E=\left\{e_{1}, e_{2}\right\}$ and

$$
\tilde{\tau}=\left\{\Phi, \tilde{X},\left(F_{1}, E\right),\left(F_{2}, E\right),\left(F_{3}, E\right)\right\}
$$

where $\Phi, \tilde{X},\left(F_{1}, E\right),\left(F_{2}, E\right),\left(F_{3}, E\right)$ are soft sets over $X$, defined as follows:

$$
\begin{aligned}
& \left(F_{1}, E\right)=\left\{\left(e_{1},\left\{x_{4}\right\}\right),\left(e_{2},\left\{x_{2}\right\}\right)\right\} \\
& \left(F_{2}, E\right)=\left\{\left(e_{1},\left\{x_{2}, x_{4}\right\}\right),\left(e_{2},\left\{x_{2}, x_{3}, x_{4}\right\}\right)\right\} \\
& \left(F_{3}, E\right)=\left\{\left(e_{1},\left\{x_{1}, x_{2}, x_{4}\right\}\right),\left(e_{2}, X\right)\right\}
\end{aligned}
$$

Then $\tilde{\tau}$ defines a soft topology on $X$ and thus $(X, \tilde{\tau}, E)$ is a soft topological space over $X$ in [10]. Clearly, $\left(F_{2}, E\right)=\left\{\left(e_{1},\left\{x_{2}, x_{4}\right\}\right),\left(e_{2},\left\{x_{2}, x_{3}, x_{4}\right\}\right)\right\}$ is a soft locally $b$-closed set in $X$ but not soft $b$-closed.

Proposition 13. Let $(F, E)$ be any soft set in a soft topological space $X$. $(F, E)$ is soft locally b-closed if and only if there exists a soft open set $(G, E)$ such that $(F, E)=(G, E) \sqcap b c l(F, E)$.

Proof. Necessity. Since $(F, E)$ is soft locally $b$-closed, $(F, E)=(G, E) \sqcap(K, E)$ where $(G, E)$ is soft open and $(K, E)$ is soft $b$-closed. Hence $(F, E) \sqsubseteq(G, E)$ and $(F, E) \sqsubseteq(K, E)$ then $(F, E) \sqsubseteq b c l(F, E) \sqsubseteq b c l(K, E)=(K, E)$. Therefore 
$(F, E) \sqsubseteq(G, E) \sqcap b c l(F, E) \sqsubseteq(G, E) \sqcap b c l(K, E)=(G, E) \sqcap(K, E)=(F, E)$. Hence, $(H, E)=(G, E) \sqcap \operatorname{bcl}(H, E)$.

Sufficiency. Since $b c l(F, E)$ is soft $b$-closed and $(F, E)=(G, E) \sqcap b c l(F, E)$, then $(F, E)$ is soft locally $b$-closed.

Theorem 14. 2] In a soft topological space $X$, every soft closed set is soft b-closed.

Now we are ready to give the relation between soft locally $b$-closed set and soft locally closed set.

Theorem 15. In a soft topological space $X$, every soft locally closed set is a soft locally b-closed set.

The following example shows that the converse implication does not hold.

Example 16. Let $X=\left\{x_{1}, x_{2}, x_{3}\right\}, E=\left\{e_{1}, e_{2}\right\}$ and

$$
\widetilde{\tau}=\left\{\Phi, \tilde{X},\left(F_{1}, E\right),\left(F_{2}, E\right),\left(F_{3}, E\right)\right\}
$$

where $\Phi, \widetilde{X},\left(F_{1}, E\right),\left(F_{2}, E\right),\left(F_{3}, E\right)$ are soft sets over $X$, defined as follows:

$$
\begin{aligned}
& \left(F_{1}, E\right)=\left\{\left(e_{1},\left\{x_{1}\right\}\right),\left(e_{2},\left\{x_{1}\right\}\right)\right\}, \\
& \left(F_{2}, E\right)=\left\{\left(e_{1},\left\{x_{2}\right\}\right),\left(e_{2},\left\{x_{2}\right\}\right)\right\}, \\
& \left(F_{3}, E\right)=\left\{\left(e_{1},\left\{x_{1}, x_{2}\right\}\right),\left(e_{2},\left\{x_{1}, x_{2}\right\}\right)\right\} .
\end{aligned}
$$

Then $\tilde{\tau}$ defines a soft topology on $X$ and thus $(X, \tilde{\tau}, E)$ is a soft topological space over $X$. Clearly, $(F, E)=\left\{\left(e_{1},\left\{x_{3}\right\}\right),\left(e_{2},\left\{x_{1}, x_{3}\right\}\right)\right\}$ is a soft locally $b$-closed set in $X$ but not soft locally closed.

Theorem 17. 2] In a soft topological space $X$,

(1) An arbitrary union of soft b-open sets is a soft b-open set.

(2) The intersection of a soft open set and a soft b-open set is a soft b-open set.

From the Theorem 17, we have the following.

Corollary 18. The intersection of a soft locally b-closed set and a soft locally closed set is soft locally b-closed.

Proposition 19. Let $X$ be a soft topological space. If $(F, E)$ is soft locally b-closed in $X$ then

(1) $b c l(F, E) \backslash(F, E)$ is a soft $b$-closed set.

(2) $[(F, E) \sqcup(\tilde{X} \backslash b c l(F, E))]$ is soft $b$-open.

(3) $(F, E) \sqsubseteq \operatorname{bint}((F, E) \sqcup(\tilde{X} \backslash b c l(F, E)))$. 
Proof. (1) If $(F, E)$ is soft locally $b$-closed, there exists a soft open set $(G, E)$ such that $(F, E)=(G, E) \sqcap b c l(F, E)$. Then

$$
\begin{aligned}
b c l(F, E) \backslash(F, E) & =b c l(F, E) \backslash[(G, E) \sqcap b c l(F, E)] \\
& =b c l(F, E) \sqcap[\tilde{X} \backslash((G, E) \sqcap b c l(F, E))] \\
& =b c l(F, E) \sqcap[(\tilde{X} \backslash(G, E)) \sqcup(\tilde{X} \backslash b c l(F, E))] \\
& =[b c l(F, E) \sqcap(\tilde{X} \backslash(G, E))] \sqcup[\operatorname{bcl}(F, E) \sqcap(\tilde{X} \backslash b c l(F, E))] \\
& =b c l(F, E) \sqcap(\tilde{X} \backslash(G, E))
\end{aligned}
$$

which is soft $b$-closed by Theorem 17 .

(2) Since $b c l(F, E) \backslash(F, E)$ is soft $b$-closed, then $[\tilde{X} \backslash(b c l(F, E) \backslash(F, E))]$ is soft $b$-open and

$$
\tilde{X} \backslash(b c l(F, E) \backslash(F, E))=\tilde{X} \backslash(b c l(F, E) \sqcap(\tilde{X} \backslash(F, E)))=(F, E) \sqcup(\tilde{X} \backslash b c l(F, E)) .
$$

(3) It is obvious that

$$
(F, E) \sqsubseteq(F, E) \sqcup(\tilde{X} \backslash b c l(F, E))=\operatorname{bint}[(F, E) \sqcup(\tilde{X} \backslash b c l(F, E))] .
$$

Theorem 20. Let $X$ be closed under finite unions of soft b-closed sets. Then the following relation hold:

$$
b c l(F, E) \sqcup b c l(G, E)=b c l((F, E) \sqcup(G, E)) .
$$

Proof. We have $(F, E) \sqsubseteq(F, E) \sqcup(G, E)$ and $(G, E) \sqsubseteq(F, E) \sqcup(G, E)$. Since $b c l(F, E) \sqsubseteq \operatorname{bcl}((F, E) \sqcup(G, E))$ and $b c l(G, E) \sqsubseteq b c l((F, E) \sqcup(G, E))$ we have $b c l(F, E) \sqcup b c l(G, E) \sqsubseteq b c l((F, E) \sqcup(G, E))[2$.

Now, $b c l(F, E)$ and $b c l(G, E)$ are soft $b$-closed sets. Then we have $(F, E) \sqcup$ $(G, E) \sqsubseteq b c l(F, E) \sqcup b c l(G, E)$ since $(F, E) \sqsubseteq b c l(F, E)$ and $(G, E) \sqsubseteq b c l(G, E)$. That is, $b c l(F, E) \sqcup b c l(G, E)$ is a soft $b$-closed set containing $(F, E) \sqcup(G, E)$. But $b c l((F, E) \sqcup(G, E))$ is the smallest soft $b$-closed set containing $(F, E) \sqcup(G, E)$. Hence $b c l((F, E) \sqcup(G, E)) \sqsubseteq b c l(F, E) \sqcup b c l(G, E)$. So, we obtain $b c l((F, E) \sqcup(G, E))=$ $b c l(F, E) \sqcup b c l(G, E)$.

The union of two soft locally $b$-closed sets is generally not soft locally $b$-closed. To define the union of two soft locally $b$-closed sets, we need to give the following concept:

Definition 21. 23] Let $X$ be a soft topological space and $(F, E),(G, E)$ are soft sets over $X .(F, E)$ and $(G, E)$ are said to be soft separated sets if $(F, E) \sqcap \operatorname{cl}(G, E)=\Phi$ and $(G, E) \sqcap \operatorname{cl}(F, E)=\Phi$. 
Theorem 22. Suppose $X$ is closed under finite unions of soft b-closed sets. Let $(F, E)$ and $(G, E)$ be soft locally b-closed. If $(F, E)$ and $(G, E)$ are soft separated, then $(F, E) \sqcup(G, E)$ is soft locally b-closed.

Proof. Since $(F, E)$ and $(G, E)$ are soft locally $b$-closed, $(F, E)=(S, E) \sqcap b c l(F, E)$ and $(G, E)=(T, E) \sqcap b c l(G, E)$, where $(S, E)$ and $(T, E)$ are soft open in $X$. $\operatorname{Put}(H, E)=(S, E) \sqcap(\tilde{X} \backslash \operatorname{cl}(G, E))$ and $(K, E)=(T, E) \sqcap(\tilde{X} \backslash c l(F, E))$. Then $(H, E) \sqcap b c l(F, E)=((S, E) \sqcap(\tilde{X} \backslash c l(G, E))) \sqcap b c l(F, E)=(F, E) \sqcap(\tilde{X} \backslash c l(G, E))=$ $(F, E)$, since $(F, E) \sqsubseteq(\tilde{X} \backslash \operatorname{cl}(G, E))$. Similarly, $(K, E) \sqcap b c l(G, E)=(G, E)$. And $(H, E) \sqcap b c l(G, E) \sqsubseteq(H, E) \sqcap c l(G, E)=\Phi$ and $(K, E) \sqcap b c l(F, E) \sqsubseteq(K, E) \sqcap$ $c l(F, E)=\Phi$. Since $(H, E)$ and $(K, E)$ are soft open, $((H, E) \sqcup(K, E)) \sqcap b c l((F, E) \sqcup$ $(G, E))=((H, E) \sqcup(K, E)) \sqcap(b c l(F, E) \sqcup b c l(G, E))=((H, E) \sqcap b c l(F, E)) \sqcup((H, E) \sqcap$ $b c l(G, E)) \sqcup((K, E) \sqcap b c l(F, E)) \sqcup((K, E) \sqcap b c l(G, E))=(F, E) \sqcup(G, E)$. We obtain $(F, E) \sqcup(G, E)$ is soft locally $b$-closed.

Lemma 23. 12 Let $X$ be a soft topological space, $(F, E)$ a soft set over $X$ and $e_{K} \in S S(X)_{E}$. Then $e_{K} \in \operatorname{pcl}(F, E)$ if and only if $(F, E) \sqcap(G, E) \neq \Phi$ for every $(G, E) \in \operatorname{SPOS}(X)$.

Lemma 24. Let $X$ be a soft topological space. If $(F, E)$ is a soft semiopen set, then $\operatorname{pcl}(F, E)=\operatorname{cl}(F, E)$.

Proof. We have $\operatorname{pcl}(F, E) \sqsubseteq \operatorname{cl}(F, E)$ for every soft set $(F, E)$ over $X$. We show that $\operatorname{cl}(F, E) \sqsubseteq \operatorname{pcl}(F, E)$ if $(F, E) \in S S O S(X)$. Let $e_{K} \in \operatorname{cl}(F, E)$ and $e_{K} \in(G, E) \in$ $S P O S(X)$, then $e_{K} \in(G, E) \sqsubseteq \operatorname{int}(\operatorname{cl}(G, E))$ and hence $(F, E) \sqcap \operatorname{int}(\operatorname{cl}(G, E)) \neq \Phi$. Since $(F, E) \in S S O S(X),(F, E) \sqcap \operatorname{int}(\operatorname{cl}(G, E)) \sqsubseteq \operatorname{cl}(\operatorname{int}(F, E)) \sqcap \operatorname{int}(\operatorname{cl}(G, E)) \sqsubseteq$ $\operatorname{cl}(\operatorname{int}(F, E) \sqcap \operatorname{cl}(G, E)) \sqsubseteq \operatorname{cl}((F, E) \sqcap(G, E))$. Therefore, we obtain $\operatorname{cl}((F, E) \sqcap$ $(G, E)) \neq \Phi$ and so $(F, E) \sqcap(G, E) \neq \Phi$. By Lemma $23, e_{K} \in \operatorname{pcl}(F, E)$ and hence $c l(F, E) \sqsubseteq \operatorname{pcl}(F, E)$.

Definition 25. A soft set $(F, E)$ in a soft topological space $X$ is called a soft $\Psi$ - set if $(F, E)=(G, E) \sqcap(K, E)$ where $(G, E)$ is soft open and $\operatorname{int}(\operatorname{cl}(K, E)) \sqsubseteq(K, E)$.

It is clear that every soft $\Psi$ - set is a soft B-set.

Theorem 26. Let $X$ be a soft topological space. A soft set $(F, E)$ over $X$ is soft $\Psi$ - set if and only if there exists a soft open set $(G, E)$ such that $(F, E)=$ $(G, E) \sqcap \operatorname{scl}(F, E)$.

Proof. Let $(F, E)$ be a soft $\Psi$ - set. Then there exists a soft open set $(G, E)$ and a soft semiclosed set $(H, E)$ such that $(F, E)=(G, E) \sqcap(H, E)$. We have $(F, E) \sqsubseteq(G, E)$, $(F, E) \sqsubseteq(H, E),(F, E) \sqsubseteq \operatorname{scl}(H, E),(F, E) \sqsubseteq(G, E) \sqcap \operatorname{scl}(F, E) \sqsubseteq(G, E) \sqcap$ $(H, E)=(F, E)$. Hence $(F, E)=(G, E) \sqcap \operatorname{scl}(F, E)$.

The converse is obvious since $\operatorname{scl}(F, E)$ is soft semiclosed.

Remark 27. Soft semiopen sets and soft locally b-closed sets are independent from each other as shown in the following examples. 
Example 28. Let $X=\left\{x_{1}, x_{2}, x_{3}, x_{4}\right\}$ and $E=\left\{e_{1}, e_{2}, e_{3}\right\}$. Let us take the soft topology $\tilde{\tau}$ on $X$ and the soft set $(F, E)=\left\{\left(e_{1}, \emptyset\right),\left(e_{2},\left\{x_{4}\right\}\right),\left(e_{3}, \emptyset\right)\right\}$ in Example 11. Clearly, $(F, E)$ is a soft locally $b$-closed set in $X$ but not soft semiopen.

Example 29. Let $X=\left\{x_{1}, x_{2}, x_{3}, x_{4}\right\}$ and $E=\left\{e_{1}, e_{2}\right\}$. Let us take the soft topology $\tilde{\tau}$ on $X$ and the soft set $(G, E)=\left\{\left(e_{1},\left\{x_{2}, x_{3}, x_{4}\right\}\right),\left(e_{2},\left\{x_{2}, x_{3}, x_{4}\right\}\right)\right\}$ in Example 12. Clearly, $(G, E)$ is a soft semiopen set in $X$ but not soft locally b-closed.

Theorem 30. Let $X$ be a soft topological space. A soft set $(F, E)$ over $X$ is a soft $B$-set if it is soft locally b-closed and soft semiopen.

Proof. Let $(F, E)$ be soft locally $b$-closed and soft semiopen. Then by Proposition 13 , there exists a soft open set $(G, E)$ such that $(F, E)=(G, E) \sqcap b c l(F, E)=$ $(G, E) \sqcap[\operatorname{scl}(F, E) \sqcap p c l(F, E)]$. By Lemma 24 , we have $(F, E)=(G, E) \sqcap[\operatorname{scl}(F, E) \sqcap$ $c l(F, E)]=(G, E) \sqcap \operatorname{scl}(F, E)$. Hence $(F, E)$ is a soft $\Psi$ - set by Theorem 26 , so $(F, E)$ is a soft B-set.

Remark 31. Soft $\alpha$-open sets and soft locally $b$-closed sets are independent from each other as shown in the following examples.

Example 32. Let $X=\left\{x_{1}, x_{2}, x_{3}, x_{4}\right\}$ and $E=\left\{e_{1}, e_{2}, e_{3}\right\}$. Let us take the soft topology $\tilde{\tau}$ on $X$ and the soft set $(F, E)=\left\{\left(e_{1}, \emptyset\right),\left(e_{2},\left\{x_{4}\right\}\right),\left(e_{3}, \emptyset\right)\right\}$ in Example 11. Clearly, $(F, E)$ is a soft locally $b$-closed set in $X$ but not soft $\alpha$-open.

Example 33. Let $X=\left\{x_{1}, x_{2}, x_{3}, x_{4}\right\}$ and $E=\left\{e_{1}, e_{2}\right\}$. Let us take the soft topology $\tilde{\tau}$ on $X$ and the soft set $(G, E)=\left\{\left(e_{1},\left\{x_{2}, x_{3}, x_{4}\right\}\right),\left(e_{2},\left\{x_{2}, x_{3}, x_{4}\right\}\right)\right\}$ in Example 12. Clearly, $(G, E)$ is a soft $\alpha$-open set in $X$ but not soft locally b-closed.

Theorem 34. Let $X$ be a soft topological space. A soft set $(F, E)$ over $X$ is soft open if and only if it is both a soft $\alpha$-open set and a soft locally b-closed set.

Proof. It is immediate from Theorem 7, Theorem 8 and Theorem 30.

Definition 35. 4] Let $X$ be a soft topological space. A soft set $(F, E)$ over $X$ is called a soft generalized b-closed set (briefly; soft gb-closed set) if bcl $(F, E) \sqsubseteq(G, E)$, whenever $(F, E) \sqsubseteq(G, E)$ and $(G, E)$ is soft open.

Theorem 36. Let $X$ be a soft topological space. A soft set $(F, E)$ over $X$ is soft $b$-closed if and only if it is both a soft gb-closed set and a soft locally b-closed set.

Proof. Necessity. Let $(F, E)$ be soft $b$-closed. $(F, E)=(F, E) \sqcap \tilde{X}$, then $(F, E)$ soft locally $b$-closed. Also, if $(F, E) \sqsubseteq(G, E)$ where $(G, E)$ is soft open, then $b c l(F, E)=(F, E) \sqsubseteq(G, E)$. Hence $(F, E)$ is soft gb-closed.

Sufficiency. If $(F, E)$ is soft locally $b$-closed, then there exists a soft open set $(G, E)$ such that $(F, E)=(G, E) \sqcap b c l(F, E)$. Since $(F, E) \sqsubseteq(G, E)$ and $(F, E)$ is soft gb-closed then $b c l(F, E) \sqsubseteq(G, E)$. Therefore $b c l(F, E) \sqsubseteq(G, E) \sqcap b c l(F, E)=$ $(F, E)$. Hence $(F, E)$ is soft $b$-closed. 


\section{Decompositions of Soft Continuity}

In this section, we introduce soft locally $b$-continuous functions and give a decomposition of soft continuity via the notion of soft locally b-closed set.

Definition 37. 13] Let $S S(X)_{E}$ and $S S(Y)_{K}$ be families of soft sets, $u: X \longrightarrow Y$ and $p: E \longrightarrow K$ be mappings. Then the mapping $f_{p u}: S S(X)_{E} \longrightarrow S S(Y)_{K}$ is defined as:

(1) Let $(F, E) \in S S(X)_{E}$. The image of $(F, E)$ under $f_{p u}$, written as $f_{p u}(F, E)=$ $\left(f_{p u}(F), p(E)\right)$, is a soft set in $S S(Y)_{K}$ such that

$$
f_{p u}(F)(y)=\left\{\begin{array}{lr}
\cup_{x \in p^{-1}(y) \cap A} u(F(x)) & , p^{-1}(y) \cap A \neq \emptyset \\
\emptyset & \text {, otherwise }
\end{array}\right.
$$

for all $y \in K$.

(2) Let $(G, K) \in S S(Y)_{K}$. The inverse image of $(G, K)$ under $f_{p u}$, written as $f_{p u}^{-1}(G, K)=\left(f_{p u}^{-1}(G), p^{-1}(K)\right)$, is a soft set in $S S(X)_{E}$ such that

for all $x \in E$.

$$
f_{p u}^{-1}(G)(x)= \begin{cases}u^{-1}(G(p(x))) & , p(x) \in K \\ \emptyset & , \text { otherwise }\end{cases}
$$

Definition 38. Let $X$ and $Y$ be soft topological spaces and $f_{p u}: S S(X)_{E} \longrightarrow$ $S S(Y)_{K}$ be a function. Then $f_{p u}$ is called

(1) soft continuous 24 if for each $(G, K) \in \operatorname{SOS}(Y), f^{-1}(G, K) \in \operatorname{SOS}(X)$.

(2) soft semicontinuous 15 if for each $(G, K) \in \operatorname{SOS}(Y), f_{p u}^{-1}(G, K) \in \operatorname{SSOS}(X)$.

(3) soft $\alpha$-continuous [1] if for each $(G, K) \in \operatorname{SOS}(Y), f_{p u}^{-1}(G, K) \in \operatorname{S} \alpha \operatorname{OS}(X)$.

(4) soft B-continuous [21] if for each $(G, K) \in \operatorname{SOS}(Y), f_{p u}^{-1}(G, K) \in \operatorname{SBS}(X)$.

(5) soft LC-continuous [14] if for each $(G, K) \in \operatorname{SOS}(Y), f_{p u}^{-1}(G, K) \in \operatorname{SLCS}(X)$.

(6) soft $b$-continuous [2] if for each $(G, K) \in \operatorname{SOS}(Y), f_{p u}^{-1}(G, K) \in \operatorname{SbOS}(X)$.

Definition 39. Let $X$ and $Y$ be soft topological spaces and $f_{p u}: S S(X)_{E} \longrightarrow$ $S S(Y)_{K}$ be a function. Then $f_{p u}$ is called soft locally b-continuous if for each $(G, K) \in S O S(Y), f_{p u}^{-1}(G, K)$ is a soft locally b-closed set in $X$.

Remark 40. Soft $\alpha$-continuous functions and soft locally b-continuous functions are independent from each other as shown in the following examples.

Example 41. Let $X=\left\{x_{1}, x_{2}, x_{3}\right\}, Y=\left\{y_{1}, y_{2}, y_{3}\right\}, E=\left\{e_{1}, e_{2}\right\}, K=\left\{k_{1}, k_{2}\right\}$, $\tilde{\tau}=\left\{\Phi, \tilde{X},\left(F_{1}, E\right),\left(F_{2}, E\right),\left(F_{3}, E\right)\right\}$ in Example 16 and $\tilde{v}=\{\Phi, \tilde{Y},(H, K)\}$ such that

$$
(H, K)=\left\{\left(k_{1},\left\{y_{1}\right\}\right),\left(k_{2},\left\{y_{1}, y_{3}\right\}\right)\right\} .
$$

Let $(X, \tilde{\tau}, E)$ and $(Y, \tilde{v}, K)$ be soft topological spaces. Define $u: X \longrightarrow Y, p$ : $E \longrightarrow K$ as 
$u\left(x_{1}\right)=\left\{y_{3}\right\}, u\left(x_{2}\right)=\left\{y_{2}\right\}, u\left(x_{3}\right)=\left\{y_{1}\right\}$ and $p\left(e_{1}\right)=\left\{k_{1}\right\}, p\left(e_{2}\right)=\left\{k_{2}\right\}$.

Let $f_{p u}: S S(X)_{E} \longrightarrow S S(Y)_{K}$ be a function. Then $(H, K)$ is soft open in $Y$ and $f_{p u}^{-1}(H, K)=\left\{\left(e_{1},\left\{x_{3}\right\}\right),\left(e_{2},\left\{x_{1}, x_{3}\right\}\right)\right\}$ is a soft locally $b$-closed set. Therefore, $f_{p u}$ is soft locally $b$-continuous. But, $f_{p u}^{-1}(H, K)$ is not a soft $\alpha$-open set and so $f_{p u}$ is not soft $\alpha$-continuous.

Example 42. Let $X=\left\{x_{1}, x_{2}, x_{3}, x_{4}\right\}, Y=\left\{y_{1}, y_{2}, y_{3}, y_{4}\right\}, E=\left\{e_{1}, e_{2}\right\}$ and $K=$ $\left\{k_{1}, k_{2}\right\} \tilde{\tau}=\left\{\Phi, \tilde{X},\left(F_{1}, E\right),\left(F_{2}, E\right),\left(F_{3}, E\right)\right\}$ in Example 12 and $\tilde{v}=\{\Phi, \tilde{Y},(G, K)\}$ such that

$$
(G, K)=\left\{\left(k_{1},\left\{y_{1}, y_{2}, y_{3}\right\}\right),\left(k_{2},\left\{y_{1}, y_{2}, y_{3}\right\}\right)\right\} .
$$

Let $(X, \tilde{\tau}, E)$ and $(Y, \tilde{v}, K)$ be soft topological spaces. Define $u: X \longrightarrow Y, p$ : $E \longrightarrow K$ as

$$
\begin{gathered}
u\left(x_{1}\right)=\left\{y_{4}\right\}, u\left(x_{2}\right)=\left\{y_{1}\right\}, u\left(x_{3}\right)=\left\{y_{2}\right\}, u\left(x_{4}\right)=\left\{y_{3}\right\} \text { and } \\
p\left(e_{1}\right)=\left\{k_{1}\right\}, p\left(e_{2}\right)=\left\{k_{2}\right\} .
\end{gathered}
$$

Let $f_{p u}: S S(X)_{E} \longrightarrow S S(Y)_{K}$ be a function. Then $(G, K)$ is soft open in $Y$ and $f_{p u}^{-1}(G, K)=\left\{\left(e_{1},\left\{x_{2}, x_{3}, x_{4}\right\}\right),\left(e_{2},\left\{x_{2}, x_{3}, x_{4}\right\}\right)\right\}$ is a soft $\alpha$-open set. Therefore, $f_{p u}$ is soft $\alpha$-continuous. But, $f_{p u}^{-1}(G, K)$ is not a soft locally $b$-closed set and so $f_{p u}$ is not soft locally $b$-continuous.

Theorem 43. Let $X$ and $Y$ be soft topological spaces. Then $f_{p u}: S S(X)_{E} \longrightarrow$ $S S(Y)_{K}$ is soft continuous if and only if it is soft $\alpha$-continuous and soft locally b-continuous.

Proof. This is an immediate consequence of Theorem 34 .

Definition 44. Let $X$ and $Y$ be soft topological spaces and $f_{p u}: S S(X)_{E} \longrightarrow$ $S S(Y)_{K}$ be a function. Then $f_{p u}$ is called soft gb-continuous if for each $(G, K) \in S O S(Y)$, $f_{p u}^{-1}(G, K)$ is a soft gb-closed set in $X$.

Theorem 45. Let $X$ and $Y$ be soft topological spaces. Then $f_{p u}: S S(X)_{E} \longrightarrow$ $S S(Y)_{K}$ is soft b-continuous if and only if it is soft gb-continuous and soft locally b-continuous.

Proof. This is an immediate consequence of Theorem 36.

\section{Conclusion}

In the present study, we have introduced soft locally $b$-closed sets in soft topological spaces which are defined over an initial universe with a fixed set of parameters and we have studied some of their properties. We have investigated their relationships with different types of subsets of soft topological spaces with the help of counterexamples. Also, the concept of soft locally $b$-continuous functions have been presented. Finally, a decomposition of soft continuity has been obtained.

In future, these findings may be extended to new types of soft sets such as soft $\alpha$ locally closed and soft pre-locally closed sets in soft topological spaces. We expect 
that results in this paper will be helpfull for further studies in soft topological spaces.

Author Contributions. All authors contributed equally and significantly in writing this paper. All authors read and approved the final manuscript.

Acknowledgement. This work was supported by Kırşehir Ahi Evran University Scientific Research Projects Coordination Unit. Project Number: FEF.A3.16.020.

\section{REFERENCES}

[1] Akdag M. and Ozkan A., Soft $\alpha$-open sets and soft $\alpha$-continuous functions, Abstract and Applied Analysis, http://dx.doi.org/10.1155/2014/891341, 2014.

[2] Akdag M. and Ozkan A., Soft b-open sets and soft b-continuous functions, Math. Sci., (2014), $8(124)$.

[3] Akdag M. and Ozkan A., On soft preopen sets and soft pre separation axioms, Gazi University Journal of Science, 27(4), (2014), 1077-1083.

[4] Al-Salem S. M., Soft regular generalized b-closed sets in soft topological spaces, Journal of Linear and Topological Algebra, 3(4), (2014), 195-204.

[5] Ali M.I., Feng F., Liu X., Min W.K. and Shabir M., On some new operations in soft set theory, Computers and Mathematics with Applications, 57, (2009), 1547-1553.

[6] Arockiarani I. and Arokialancy A., Generalized soft $\mathrm{g} \beta$-closed sets and soft gs $\beta$-closed sets in soft topological spaces, International Journal of Mathematical Archive, 4(2), (2013), 1-7.

[7] Aygünoğlu A. and Aygün H., Some notes on soft topological spaces, Neural Computing and Applications, 21(1), (2012), 113-119.

[8] Chen B., Soft semi-open sets and related properties in soft topological spaces, Applied Mathematics 83 Information Sciences, 7(1), (2013), 287-294.

[9] Feng F., Jun Y.B. and Zhao X.Z., Soft semirings, Computers and Mathematics with Applications, 56, (2008), 2621-2628.

[10] Guzel Ergul Z. and Tozlu N., Decompositions of soft continuity and soft AB-continuity, Annals of Fuzzy Mathematics and Informatics, 14(5), (2017), 463-474.

[11] Hussain S. and Ahmad B., Some properties of soft topological spaces, Computers and Mathematics with Applications, 62(11), (2011), 4058-4067.

[12] Kandil A., Tantawy O.A.E., El-Sheikh S.A. and Abd El-latif A.M., $\gamma$-operation and decompositions of some forms of soft continuity in soft topological spaces, Ann. Fuzzy Math. Inform., $7(2),(2014), 181-196$.

[13] Kharal A. and Ahmad B., Mappings on soft classes, New Mathematics and Natural Computation, 7(3), (2011), 471-481.

[14] Kocaman A.H. and Tozlu N., Soft locally closed sets and decompositions of soft continuity, Annals of Fuzzy Mathematics and Informatics, 11(2), (2016), 173-181.

[15] Mahanta J. and Das P.K., On soft topological space via semi-open and semi-closed soft sets, Cornell University Library, arXiv:1203.4133, 2012.

[16] Maji P.K., Roy A.R. and Biswas R., An application of soft sets in decision making problem, Computers and Mathematics with Applications, 44, (2002), 1077-1083.

[17] Maji P.K., Biswas R. and Roy A.R., Soft set theory, Computers and Mathematics with Applications, 45(4-5), (2003), 555-562.

[18] Molodtsov D., Soft set theory-first results, Computers and Mathematics with Applications, 37(4-5), (1999), 19-31.

[19] Molodtsov D., Leonov V.Y. and Kovkov D.V., Soft sets technique and its application, Nechetkie Sistemy i Myagkie Vychisleniya, 9(1), (2006), 8-39. 
[20] Shabir M. and Naz M., On soft topological spaces, Computers and Mathematics with Applications, 61(7), (2011), 1786-1799.

[21] Tozlu N. and Yüksel Ş., Soft A-sets and soft B-sets in soft topological spaces, Mathematical Sciences 8 Applications E- Notes, 5(2), (2017), 17-25.

[22] Yüksel Ş., Tozlu N. and Güzel Ergül Z., Soft regular generalized closed sets in soft topological spaces, International Journal of Mathematical Analysis, 8(8), (2014), 355-367.

[23] Yuksel S., Guzel Ergul Z. and Guven Z., Soft connected spaces, International J. of Pure 8 Engg. Mathematics, 2(3), (2014), 121-134.

[24] Zorlutuna I., Akdağ M., Min W.K. and Atmaca S., Remarks on soft topological spaces, Annals of Fuzzy Mathematics and Informatics, 3(2), (2012), 171-185.

Current address: Naime Demirtaş: Mersin University, Science and Art Faculty, Department of Mathematics, Mersin, TURKEY

E-mail address: naimedemirtas@mersin.edu.tr

ORCID Address: http://orcid.org/0000-0003-4137-4810

Current address: Zehra Güzel Ergül (Corresponding author): Kırsehir Ahi Evran University, Science and Art Faculty, Department of Mathematics, Kırşehir, TURKEY

E-mail address: zguzel@ahievran.edu.tr

ORCID Address: http://orcid.org/0000-0001-5717-1626 